\title{
INCIDÊNCIA DE CEFALÉIA EM UMA COMUNIDADE HOSPITALAR
}

\author{
VIVIANE H. FLUMIGNAN ZÉTOLA*, EDISON MATOS NÓVAK**, ANDRÉA LUIZ***, \\ BENEDITO O. DE S. BRANCO***, BERENICE KOMINEK SATO***, CRISTINA SAYURI NITA***, \\ MARCOS HENRIQUE BUBNA ***, RODRIGO DE ALMEIDA PRADO***, LINEU CESAR WERNECK ****
}

RESUMO - O objetivo foi determinar em um grupo de pessoas de uma comunidade hospitalar a incidência de cefaléia e para esta a frequência, principais características e investigações médicas mais solicitadas. Utilizamos a combinação de questionário e entrevista. Do total de 1006 fichas aleatoriamente preenchidas, 987 pessoas responderam corretamente aos quesitos e destas 380 (38,5\%) eram portadoras de cefaléia. Baseados na Classificação Internacional de Cefaléia dividimos os portadores em dois principais grupos, a migrânea e a cefaléia do tipo tensional. As demais foram agrupadas num terceiro grupo. A idade média foi 31,18 anos, com predomínio do sexo feminino em todos os tipos de cefaléia. A presença de história familiar foi positiva em $76,8 \%$ dos entrevistados. As características mais frequentes foram: localização frontal, tipo pulsátil e intensidade moderada. O principal fator desencadeante foi o estresse. A procura de acompanhamento médico deu-se em $41,3 \%$ dos portadores. Destes, aproximadamente 56\% consultaram um clínico geral, $23 \%$ consultaram um neurologista e $21 \%$ procuraram outras especialidades. O RX de crânio foi o exame mais solicitado pelos generalistas e o eletrencefalograma pelos neurologistas. A tomografia computadorizada do crânio não foi solicitada com frequência.

PALAVRAS-CHAVE: cefaléia, migrânea, estresse.

\section{Headache incidence in a hospital community}

ABSTRACT - The purpose was to describe the main features of headache incidence in a hospital community, its frequency and the most requested medical investigation. Due to the stressful work environment, hospital is considered to hold a high-risk population. Interviews and questionnaires were utilized. Of a 1006 files, which were randomly filled out, 987 could be analyzed. Of all, 38,5\% were from headache sufferers. By using a table of pain symptoms taken from the International Headache Society classification as a pattern, headaches were assigned as migraine, tension-type and other. The mean age was 31.18 and the frequency in females was higher than in males, at any type. Family occurrence in first-degree relatives was $76.8 \%$. Frontal location, medium intensity and pulsation were the most described features. Stress was the most frequently mentioned trigger factor. A physician was consulted only by $41.3 \%$. Cranium X-ray was the most frequently requested exam.

KEY WORDS: migraine, headache, stress.

A cefaléia é um dos sintomas mais frequentes vistos por um clínico geral, representando a queixa mais usual num ambulatório médico, a partir de onde estima-se a sua prevalência e cujos dados sugerem que $80 \%$ da população irá sofrer de dor de cabeça numa dada fase de sua vida. As estimativas de prevalência variam muito entre os estudos, sendo que especificamente a migrânea pode afetar até $18 \%$ da população feminina e $8 \%$ da população masculina ${ }^{1}$.

Estudos epidemiológicos de cefaléias têm sido realizados com intuito de reconhecer e aprimorar suas características, contribuindo para o melhor entendimento fisiopatológico desta entidade. Estudos

Disciplina de Neurologia do Departamento de Clínica Médica da Universidade Federal do Paraná (UFPR), Curitiba, Brasil: *Neurologista; **Professor Adjunto; ***Médico; ****Professor Titular. Aceite: 20-maio-1998.

Dr. Edison Matos Nóvak - Serviço de Neurologia, Hospital de Clínicas - Rua General Carneiro 181, $12^{\circ}$ andar 80060-900 Curitiba PR - Brasil. 
de prevalência da migrânea têm sugerido fatores de risco genéticos e sociodemográficos ${ }^{2}$. Dados de diversas populações têm sido levantados, mas uma população que tem recebido pouca atenção é a comunidade de trabalho hospitalar. Considerando diversas teorias etiopatogênicas da dor de cabeça, o fator estresse tem sido citado com frequência como fator "gatilho" para a cefaléia, seja do tipo tensional ou mesmo a migrânea ${ }^{3-5}$. Esse dado nos levou a considerar a importância da incidência de cefaléia dentro de um ambiente considerado estressante, ou seja, o ambiente hospitalar.

\section{CASUÍSTICA E MÉTODOS}

Foram entrevistados aleatoriamente 1006 funcionários do Hospital de Clínicas da UFPR no período de setembro a dezembro de 1993, utilizando uma combinação de entrevista e questionário. Incluíram-se médicos, estudantes, enfermeiros, técnicos, auxiliares, burocratas e assistentes sociais, entre os principais. A pesquisa foi realizada no ambiente de trabalho. Devido ao preenchimento incompleto ou incorreto, analisaram-se 987 fichas.

Utilizamos uma tabela de sintomas de dor e outras características que foi baseada em classificação de cefaléia da Sociedade Internacional de Cefaléia (International Headache Society - IHS) ${ }^{6}$. Optamos por codificar a cefaléia com os critérios diagnósticos que se utilizam de um a dois dígitos no tratamento estatístico dos dados. As cefaléias foram ordenadas de acordo com dois grupos principais, a migrânea e a cefaléia do tipo tensional. As demais cefaléias classificadas em outros tipos, incluindo as não classificáveis, foram agrupadas em "outras". Observamos a presença de mais de um tipo de cefaléia num mesmo indivíduo. Utilizamos a mais importante forma indicada pelo próprio entrevistado para classificar a cefaléia. Os entrevistados foram instruídos a definir a intensidade da dor de cabeça conforme "escore de incapacidade de trabalho": leve, dificulta as atividades de vida diárias; moderada, limita as atividades de vida diárias, e severa, impede as atividades de vida diárias.

Do total de indivíduos, $380(38,5 \%)$ referiram ser portadores de cefaléia, sendo que nestes houve predomínio do sexo feminino (257:123). A idade média foi 31,18 anos $( \pm 10,35)$, e 26,60 anos $( \pm 9,74)$ para os não-portadores. A Tabela 1 mostra a relação da presença de cefaléia e a função exercida. Para fins de apreciação apresentamos também o total de entrevistados sem cefaléia.

Tabela 1. Função x tipos de cefaléia.

\begin{tabular}{|c|c|c|c|c|c|c|}
\hline \multirow[b]{2}{*}{ Entrevistados } & \multicolumn{3}{|c|}{ Cefaléia } & \multicolumn{2}{|c|}{ Tipos de cefaléia } & \multirow[b]{2}{*}{ outros } \\
\hline & $\mathrm{n}^{\circ}$ & sem & com & migrânea & tensional & \\
\hline Assistente social & 8 & 4 & 4 & 4 & - & - \\
\hline Técnico de enfermagem & 150 & 93 & 57 & 31 & 23 & 3 \\
\hline Auxiliar administrativo & 104 & 48 & 56 & 32 & 21 & 3 \\
\hline Cozinheiro & 11 & 7 & 4 & 1 & 3 & - \\
\hline Enfermagem & 39 & 20 & 19 & 12 & 5 & 2 \\
\hline Estudante & 425 & 326 & 99 & 83 & 16 & - \\
\hline Farmacêutico & 23 & 8 & 15 & 7 & 7 & 1 \\
\hline Auxiliar de lavanderia & 30 & 6 & 24 & 8 & 15 & 1 \\
\hline Auxiliar de manutenção & 6 & 3 & 3 & 1 & 1 & 1 \\
\hline Médico & 75 & 43 & 32 & 25 & 5 & 2 \\
\hline Nutricionista & 9 & 3 & 6 & 6 & - & - \\
\hline Outros* & 7 & 5 & 2 & 1 & 1 & - \\
\hline Técnico laboratório & 71 & 23 & 48 & 33 & 13 & 2 \\
\hline Técnico RX & 7 & 5 & 2 & 1 & 1 & - \\
\hline Zelador & 22 & 13 & 9 & 4 & 4 & 1 \\
\hline Total & 987 & 607 & 380 & 249 & 115 & 16 \\
\hline
\end{tabular}

*Psicólogo, desenhista, jornalista, necropsista, técnico de ECG, telefonista. 


\section{RESULTADOS}

Do total dos entrevistados que apresentavam história de dor de cabeça crônica, 249 (65,5\%) foram classificados como portadores de migrânea; 105 (27,6\%) como cefaléia tipo tensional e 26 $(6,8 \%)$ em outros grupos. O sexo feminino foi o predominante para todos os tipos de cefaléia, sendo respectivamente 169:80, 72:33 e 16:10 para a migrânea, tensional e outros grupos diagnósticos. Não houve diferença significante entre as médias de idade nos diferentes tipos de dor de cabeça, sendo 31,03 $( \pm 10,65)$ para a migrânea, $31,95( \pm 9,17)$ para a cefaléia tensional e $29,61( \pm 12,02)$ para as outras.

Mostramos relação entre a função e os tipos de cefaléia na Tabela 1.

Quando questionados quanto a presença de história familiar, 76\% dos entrevistados responderam positivamente, sendo a herança materna predominante em todos os tipos: $63,8 \%$ para a migrânea, $29,1 \%$ para a tensional e $7,1 \%$ para as pertencentes a outros grupos. Em relação às principais características, $26,3 \%$ foram consideradas de intensidade severa, 48,9\% moderada e 24,7\% leve. Houve predominância da intensidade "severa" na migrânea.

Todos os que referiram caráter pulsátil preencheram os critérios de migrânea; nesses, apenas $4 \%$ a descrevaram como constante, $1 \%$ em queimação e $2 \%$ não soube definir. Dos portadores de cefaléia tensional, $85 \%$ descreveram como aperto/pressão e $15 \%$ como em queimação. Todas as cefaléias classificadas em outros grupos diagnósticos não definiram sua característica principal.

A Tabela 2 mostra a frequência das principais localizações e suas relações com o tipo de cefaléia.

Em relação aos sintomas premonitórios, a principal queixa foi "indisposição" (30,3\%), seguida de dor fraca inicial $(22,6 \%)$, fotofobia $(15,3 \%)$ e borramento visual $(13,2 \%)$. Os pacientes que apresentaram escotomas, diplopia e "flash" de luz foram todos relacionados a migrânea. A parestesia foi relatada como sintoma associado por apenas $10(2,63 \%)$ dos entrevistados, sendo que 8 destes eram portadores de migrânea. Vertigem ocorreu em $13,2 \%$, e em sua maioria (82\%) acompanhou a migrânea. Embora em menor frequência, parestesias e borramento visual também estiveram presentes na cefaléia tensional ( $2 \%$ e $4 \%$ respectivamente).

Quanto à duração da dor, 49,5\% referiram período entre 2 e 24 horas e apenas $13,4 \%$ referiram entre 24 e 72 horas, em ambos havendo predomínio na migrânea.

Os sintomas mais frequentemente associados foram a presença de náuseas/vômitos e fonofobia/ fotofobia, em $80 \%$ das vezes relacionados a migrânea, em que a associação com hemiplegia,

Tabela 2. Localização da dor x tipo de cefaléia.

\begin{tabular}{lcccc}
\hline & \multicolumn{3}{c}{ Tipo de cefaléia } & \\
\cline { 2 - 4 } Localização & Migrânea & Tensional & Outros & Total \\
\hline Holocraniana & $22(64,7 \%)$ & $12(35,3 \%)$ & & $34(8,9 \%)$ \\
Hemicrania constante & $24(85,7 \%)$ & $3(10,7 \%)$ & $1(3,6 \%)$ & $28(7,4 \%)$ \\
Hemicrania alternante & $9(69,2 \%)$ & $2(15,4 \%)$ & $2(15,4 \%)$ & $13(3,4 \%)$ \\
Vértex & $5(71,4 \%)$ & $2(28,6 \%)$ & & $7(1,8 \%)$ \\
Frontal & $75(62,5 \%)$ & $40(33,3 \%)$ & $5(4,2 \%)$ & $120(31,6 \%)$ \\
Temporal & $41(71,9 \%)$ & $9(15,8 \%)$ & $7(12,3 \%)$ & $57(15,0 \%)$ \\
Cervical/occipital & $11(37,9 \%)$ & $17(58,6 \%)$ & $1(3,4 \%)$ & $29(7,6 \%)$ \\
Ocular/orbitária & $26(72,2 \%)$ & $9(25,0 \%)$ & $1(2,8 \%)$ & $36(9,5 \%)$ \\
Mais de uma & $36(64,2 \%)$ & $11(19,6 \%)$ & $9(16,07 \%)$ & $56(14,7 \%)$ \\
\hline
\end{tabular}


Tabela 3. Fatores desencadeantes X Tipos de cefaléia.

\begin{tabular}{lcccc}
\hline & \multicolumn{3}{c}{ Tipo de cefaléia } & \\
\cline { 2 - 4 } Fator & Migrânea & Tensional & Outros & Total \\
\hline Luz forte & $47(77 \%)$ & $10(16,4 \%)$ & $4(6,6 \%)$ & $61(16,1 \%)$ \\
Barulho & $72(74,2 \%)$ & $21(21,6 \%)$ & $4(4,1 \%)$ & $97(25 \%)$ \\
Estresse & $180(69 \%)$ & $69(26,4 \%)$ & $12(4,6 \%)$ & $261(68,7 \%)$ \\
Jejum & $56(73,7 \%)$ & $16(21,1 \%)$ & $4(5,3 \%)$ & $76(20 \%)$ \\
Distúrbios do sono & $67(67,7 \%)$ & $27(27,3 \%)$ & $5(5,1 \%)$ & $99(26,1 \%)$ \\
Exercício físico & $12(63,2 \%)$ & $6(31,6 \%)$ & $1(5,3 \%)$ & $19(5 \%)$ \\
Fumo & $24(72,7 \%)$ & $8(24,2 \%)$ & $1(3 \%)$ & $33(8,7 \%)$ \\
Alteração climática & $23(74,2 \%)$ & $7(22,6 \%)$ & $1(3,2 \%)$ & $31(8,2 \%)$ \\
Menstruação & $43(58,1 \%)$ & $24(32,4 \%)$ & $7(9,5 \%)$ & $74(19,5 \%)$ \\
Álcool & $36(73,5 \%)$ & $10(20,4 \%)$ & $3(6,1 \%)$ & $49(12,9 \%)$ \\
Alimentação & $28(77,8 \%)$ & $6(16,7 \%)$ & $2(5,6 \%)$ & $36(9,5 \%)$ \\
Ausência de fatores & $14(63,6 \%)$ & $6(27,3 \%)$ & $2(9,1 \%)$ & $22(5,8 \%)$ \\
\hline
\end{tabular}

hemianopsia, disfasia e diplopia foram exclusivas. Outras alterações visuais foram associadas a este tipo de cefaléia em $90 \%$.

O principal fator desencadeante relatado pelos entrevistados foi o estresse, conforme mostrado na tabela 3.

Não houve relação significativa entre o tipo de cefaléia e o período de aparecimento da dor. O período da tarde foi principalmente relatado por 137 (36\%) dos portadores. A ocorrência ao despertar foi citada por apenas $7 \%$ dos entrevistados, em 74,1\% destes relacionado a migrânea.

Os principais fatores de alívio relatados foram o repouso e o uso de medicação por $48,2 \%$ e $49,7 \%$ dos entrevistados, respectivamente. O sono foi mencionado como fator de alívio por apenas $26,3 \%$ do total.

Do total dos entrevistados, $223(58,6 \%)$ não procuraram assistência médica, $88(23,2 \%)$ haviam consultado ou faziam acompanhamento com clínico geral, 36 (9,5\%) com neurologista e $33(8,68 \%)$ com médicos de outras especialidades.

Tabela 4. Exames complementares x tipos de cefaléia.

\begin{tabular}{lcccc}
\hline & \multicolumn{3}{c}{ Tipo de cefaléia } & \\
\cline { 2 - 4 } Exame & Migrânea & Tensional & Outros & Total \\
\hline Eletroencefalograma & $30(81,1 \%)$ & $5(13,5 \%)$ & $2(5,4 \%)$ & $37(9,7 \%)$ \\
Tomografia de crânio & $20(90,9 \%)$ & $1(4,5 \%)$ & $1(4,5 \%)$ & $22(5,8 \%)$ \\
RX de crânio & $35(79,5 \%)$ & $8(18,2 \%)$ & $1(2,3 \%)$ & $44(11,6 \%)$ \\
Líquido cefalorraquidiano & $3(1,2 \%)$ & & & $3(0,8 \%)$ \\
Hemograma & $11(64,7 \%)$ & $5(29,4 \%)$ & $1(5,9 \%)$ & $17(4,5 \%)$ \\
Exame oftalmológico & $32(78,0 \%)$ & $6(14,6 \%)$ & $3(7,3 \%)$ & $41(10,8 \%)$ \\
Outros exames & $3(50,0 \%)$ & $3(50,0 \%)$ & & $6(1,6 \%)$ \\
Sem exames & $110(60,8 \%)$ & $7(31,5 \%)$ & $14(7,7 \%)$ & $181(47,6 \%)$ \\
\hline
\end{tabular}


A Tabela 4 mostra os exames complementares mais solicitados na investigação da cefaléia, relacionados ao tipo de cefaléia. O RX de crânio foi o exame mais solicitado pelos generalistas e o eletrencefalograma pelos neurologistas. A tomografia computadorizada do crânio não foi solicitada com frequência.

\section{DISCUSSÃO}

Após a definição dos critérios diagnósticos pela $\mathrm{IHS}^{6}$, vários estudos epidemiológicos têm sido realizados, observando-se alta prevalência da cefaléia ${ }^{2,7,8}$. Embora os mecanismos fisio e etiopatogênicos sejam distintos (e indefinidos) nas diversas formas de apresentação da cefaléia, esta prevalece nas mulheres e em indivíduos entre 25 e 55 anos $^{8,9}$. Estudos falham em caracterizar um modo de transmissão gênica ${ }^{10}$ (exceto a sugestão de hereditariedade no subgrupo das cefaléias vasculares) ou mesmo uma clara evidência de validade fenotípica ${ }^{4,11}$. Parece certo, no entanto, que alguns fatores ambientais como o estresse atuam como "gatilhos" nas principais formas de cefaléia: migrânea e tipo tensional ${ }^{4,12,13}$. Estudos longitudinais são realizados buscando determinar a história natural das cefaléias, conhecendo suas co-morbidades ${ }^{14}$. Embora a migrânea seja uma causa conhecida de incapacidade temporária ${ }^{5,12,14}$, muitos portadores nunca consultaram um médico para o problema.

Nosso estudo demonstrou alta incidência de cefaléia na população de trabalho hospitalar, coincidindo com a literatura quanto a faixa etária e a maior frequência no sexo feminino para todos os subtipos. Esse alto nível deve-se em parte ao fato de incluir a análise estatística todos os subtipos de cefaléia. Quando separada por grupos principais, diferentemente de outras populações, encontramos maior frequência de migrânea em relação às classificadas como do tipo tensional, que usualmente figuram em maior número. A análise do tipo de função exercida demonstrou que tanto os que participam do atendimento direto de doentes, quanto as equipes de apoio burocrático ou técnico, bem como estudantes ou médicos, sofrem dessa desordem numa percentagem mínima de $23 \%$ dos entrevistados, donde se sugere que o ambiente hospitalar como um todo pode ser considerado fator estressante. Estudo anterior, de análise entre os estudantes de medicina, também registrou alta prevalência ${ }^{15}$. No presente estudo o tipo de cefaléia mais comum na nossa população foi a migrânea, não diferindo entre os profissionais das diversas repartições. $\mathrm{O}$ estresse foi fator desencadeante comum e o mais frequentemente citado, como também referido por outros autores ${ }^{5}$.

Embora em ambiente médico, com facilidades de acesso a consultas e mesmo a exames complementares, nossa amostra revelou pouca procura de acompanhamento. Não se verificou abuso na solicitação de exames de alto custo como a tomografia computadorizada de crânio, embora o eletrencefalograma tenha sido solicitado em demasia na investigação.

Nosso questionário de pesquisa não explorou especificamente motivações relacionadas ao trabalho, relações interpessoais ou saúde mental. Estudos específicos são necessários para maior embasamento conceitual e metodológico dos modelos explicativos da relação entre o estresse e ocorrência da cefaléia. Informações adicionais são também necessárias, como a análise detalhada sobre o curso da cefaléia e o período de trabalho com exclusões de outros estressores, ou ainda sobre a estatística comparativa entre grupos controle.

Nossa análise preliminar abre o caminho para a continuidade do estudo dentro dessa importante comunidade de trabalho, pois a alta prevalência de cefaléia pode estar relacionada ao estresse psicossocial, particularmente ao estresse do trabalho.

\section{REFERÊNCIAS}

1. Silberstein SD, Lipton RB. Headache epidemiology. Neurol Clin 1996;14:421-434.

2. Stewart WF, Simon D, Shechter A, Lipton RB. Population variation in migraine prevalence: a meta-analysis. J Clin Epidemiol 1995;48:269-280.

3. Martin PR, Nathan PR. Differential prevalence rates for headaches: a function of stress and social support? Headache 1987;27:329-333.

4. Robbins L. Precipitating factors in migraine: a retrospective review of 494 patients. Headache 1994;34:214-216. 
5. Antonov K, Isacson D. Headache in Sweden: the importance of working conditions. Headache 1997;37:228-234.

6. Headache Classification Committee of the International Headache Society. Classification and diagnostic criteria for headache disorders, cranial neuralgia and facial pain. Cephalalgia 1988;8:Suppl 7.

7. Stewart WF, Lipton RB, Celentano DD, Reed ML. Prevalence of migraine headache in the United States: relation to age, income, race, and other sociodemographic factors. JAMA 1992;267:64-69.

8. Lipton RB, Stewart WF. The epidemiology of migraine. Eur Neurol 1994,34 (Suppl 2):6-11.

9. Nadaoka T, Kanda H, Oiji A, Morioka Y, Kashiwakura M, Totsuka S. Headache and stress in a group of nurses and government administrators in Japan. Headache 1997;37:386-391.

10. Merikangas KR. Genetics of migraine and other headache. Curr Opin Neurol 1996:9:202-205.

11. Mauskop A, Altura BT, Cracco RQ, Altura BM. Chronic daily headache-one disease or two? Diagnostic role of serum ionized magnesium. Cephalalgia 1994;14:24-28.

12. Edmeads J, Findlay H, Tugwell P, Pryse-Phillips W, Nelson RF, Murray TJ. Impact of migraine and tension-type headache on life-style, consulting behavior and medication use: a Canadian population survey. Can J Neurol Sci 1993;20:131-137.

13. Tekle Haimanot F, Seraw B, Forsgren L, Ekbom K, Ekstedt J. Migraine, Chronic-type headache, and cluster headache in an Ethiopian rural community. Cephalalgia 1995;15:482-488.

14. Pryse-Phillips W, Findlay F, Tugwell P, Edmeads J, Murray TJ, Nelson RF. A Canadian population survey on the clinical, epidemiologic and societal impact of migraine and tension-type headache. Can J Neurol Sci 1992;19:333-339.

15. Sanvito WL, Monzillo PH, Peres MF, et al. The epidemiology of migraine in medical students. Headache 1996;36:316-319. 